\title{
Achieving and sustaining herd immunity to SARS-CoV-2
}

\author{
Shelly Bolotin MSc PhD, Sarah Wilson MSc MD, Michelle Murti MPH MD
}

Cite as: CMAJ 2021 July 19;193:E1089. doi: 10.1503/cmaj.210892

1 s Herd immunity provides indirect protection to people who are susceptible to an infectious disease

The herd immunity threshold is the minimum proportion of the population that must be immune to an infectious disease, usually due to vaccination, for the incidence of the disease to remain stable or decrease. ${ }^{1}$

\section{The herd immunity threshold correlates with the infectiousness of the pathogen}

The herd immunity threshold for measles, one of the most infectious diseases, is about $94 \%{ }^{1}$ For SARS-CoV-2 variants of concern, such as B.1.1.7 (Alpha), the threshold is about $80 \%^{2}$ and it may be higher for newly emerging variants like B.1.617.2 (Delta). ${ }^{3}$

\section{Effective vaccines with lasting protection are essential for achieving herd immunity}

Vaccines are not perfect, and some vaccinated people can still become infected and transmit SARS-CoV-2. ${ }^{4}$ People who are immunosuppressed may not mount an adequate immune response. Outbreaks may occur despite high vaccine coverage in the population. Effectiveness varies by type of vaccine, number of doses administered and the particular SARS-CoV-2 variant in circulation. Based on estimates of the effectiveness of 2 vaccine doses against symptomatic infection with B.1.1.7 (Alpha) in Ontario (90\% effectiveness), nearly $90 \%$ vaccine coverage in the population may be required for herd immunity. ${ }^{5}$ Increases in the prevalence of more infectious variants, and reduced vaccine effectiveness against them, would require even higher coverage. The duration of immunity from infection or vaccination is currently unknown. ${ }^{4}$

\section{Vaccine hesitancy and structural barriers to vaccination may threaten herd immunity}

High and even vaccine coverage is needed to achieve herd immunity. Achieving sufficient vaccine coverage depends on addressing vaccine hesitancy and barriers to vaccination that may result in pockets of susceptibility.

\section{5}

\section{Maintaining herd immunity requires sustained effort}

Maintaining herd immunity will depend on vaccines being effective against variants, addressing barriers to vaccination, and sustaining coverage if repeat vaccination is required and as the population changes (e.g., due to births, immigration). Even if herd immunity is not achieved, high vaccine coverage will substantially reduce rates of morbidity and mortality, and lessen the burden of COVID-19 on Canada's health care systems.

\section{References}

1. Vynnycky E, White RG. An introduction to infectious disease modelling. Oxford (UK): Oxford University Press; 2010.

2. Hodgson D, Flasche S, Jit M, et al.; Centre for Mathematical Modelling of Infectious Disease (CMMID) COVID-19 Working Group. The potential for vaccination-induced herd immunity against the SARS-CoV-2 B.1.1.7 variant. Euro Surveill 2021;26:2100428.

3. 3 June 2021 risk assessment for SARS-CoV-2 variant: Delta (VOC-21APR-02, B.1.617.2). London (UK): Public Health England; 2021. Available: https://www.gov.uk/government/publications /investigation-of-sars-cov-2-variants-of-concern-variant-risk -assessments (accessed 2021 June 8).

4. Phillips N. The coronavirus is here to stay - here's what that means. Nature 2021;590:382-4.

5. Chung $\mathrm{H}, \mathrm{He} \mathrm{S}$, Nasreen S, et al. Effectiveness of BNT162b2 and mRNA-1273 COVID-19 vaccines against symptomatic SARS CoV-2 infection and severe COVID-19 outcomes in Ontario, Canada. medRxiv 2021 May 28. doi: 10.1101/2021.05.24.21257744.

Competing interests: Shelly Bolotin reports funding from the Canadian Institutes of Health Research, the Canadian Immunization Research Network, the COVID-19 Immunity Task Force and the Public Health Agency of Canada, outside the submitted work. She is a member of the Canadian Immunization Research Network Management Committee, COVID-19 Immunity Task Force Leadership Group. The authors are employees of Public Health Ontario; this organization paid for the article processing fees. No other competing interests were declared.

This article has been peer reviewed.

Affiliations: Public Health Ontario, and Dalla Lana School of Public Health, University of Toronto, Toronto, Ont.

Content licence: This is an Open Access article distributed in accordance with the terms of the Creative Commons Attribution (CC BY-NC-ND 4.0) licence, which permits use, distribution and reproduction in any medium, provided that the original publication is properly cited, the use is noncommercial (i.e., research or educational use), and no modifications or adaptations are made. See: https://creativecommons. org/licenses/by-nc-nd/4.0/

Correspondence to: Shelly Bolotin, shelly.bolotin@oahpp.ca 